\title{
Remote-controlled photonics laboratories for distance learning
}

David Lieberman, Tak Cheung

David H. Lieberman, Tak D. Cheung, "Remote-controlled photonics laboratories for distance learning," Proc. SPIE 4588, Seventh International Conference on Education and Training in Optics and Photonics, (28 May 2002); doi: $10.1117 / 12.468714$

SPIE Event: Education and Training in Optics and Photonics 2001, 2001, Singapore, Singapore 


\title{
Remote-controlled photonics laboratories for distance learning
}

\author{
David Lieberman and Tak Cheung \\ Queensborough Community College \\ Bayside, NY
}

\begin{abstract}
At Queensborough Community College, with the support of the National Science Foundation (Advanced Technological Education grant award \#DUE - 9752061), we have addressed the issues of distance learning and laboratories and are adapting courses for our Laser and Fiber-Optics Technology Program for distance learning. The "problem" of the laboratory is solved by remote-controlled laboratory equipment. We have completed the work on course materials in physical optics, lasers and fiber optics. Course materials include interactive multimedia textbooks and laboraotry manuals along with the remote-controlled laboratory exercises. The remote-controlled exercises are "real" experiments with "real' data as opposed to simulations. The real nature of the exercises allows for the unexpected, which occurs in any laboratory situation. Remote-controlled laboratory exercises include interferometry, diffraction, polarization, acousto-optics, electro-optics, second harmonic generation, Q-switching, modelocking, thermal lensing, diode laser characteristics, laser principles, optical time domain reflectometry, coupling losses, wave division multiplexing and characteristics of fiber optic switches and couplers. As course materials were developed they were tested at a remote site, Suffolk County Community College.
\end{abstract}

Keywords: Distance learning, laboratory, education, remote-control, photonics, lasers, fiber optics

\section{INTRODUCTION}

As technology has advanced, the demand for qualified scientists, engineers and technicians has increased dramatically. Institutions of higher learning have not been able to keep up with this demand. In photonics, this situation will not change in the near future ${ }^{1}$. To meet the ever-increasing demand for technicians in photonics, access to current technology programs must be expanded along with the introduction of programs at more colleges and universities, a difficult task to accomplish. At Queensborough Community College (QCC) we have trained technicians in photonics through our Laser and Fiber Optics Technology (LFOT) Program since 1987. Since the program's inception we have had to work hard to maintain enrollment. Student demand for programs in photonics is small because photonics is not a popular choice of high school graduates. Therefore, a program needs to draw students from a large geographical area. The problem is that most students who attend two-year colleges live at home and do not want to spend an appreciable amount of time commuting. One way to reach a broad geographic area is to have many institutions offer programs. The major obstruction to the development of photonics technology programs is the cost. Even if the number of potential students warranted the establishment of a program, the start-up costs are prohibitive.

One way to spread a wider net is through distance learning. Distance learning gives access to students over a wider area. It also allows the sharing of resources by allowing students at one institution to take courses at another institution. Although distance learning in various forms is not new, until recently laboratory courses required the student to be physically present. Recent advances are changing the situation. We at QCC were able to develop the LFOT program because we received substantial external funding. This funding included four National Science Foundation (NSF) Improvement in Laboratory Instrumentation (ILI) grants, DUE-90-52219, DUE-91-52109, DUE-93-51683 and DUE-9650617. Realizing that other institutions do not have neither the equipment nor the resources to acquire it, we at QCC with the support of the NSF, through an Advanced Technological Education grant, DUE - 9752061, have developed materials and methods to provide photonics technology education at a distance.

There are three specialized courses in our LFOT curriculum which require extensive instrumentation in the laboratory. The subject areas of the courses are; lasers and detectors, fiber optics and physical optics including modulators and a brief introduction to non-linear optics. Each course has lecture and laboratory components both of which are addressed in conversion to distance learning. Course materials include interactive multimedia textbooks and laboratory manuals along with the remote-controlled laboratory exercises. Remote-controlled laboratory exercises include interferometry,

Seventh International Conference on Education and Training in Optics and Photonics, edited by Tuan-Kay Lim, Arthur H. Guenther, Proc. of SPIE Vol. 4588, · (c) 2010 SPIE · CCC code: 0277-786X/10/\$18 · doi: 10.1117/12.468714 
diffraction, polarization, acousto-opotics, electro-optics, second harmonic generation, Q-switching, modelocking, thermal lensing, diode laser characteristics, laser principles, optical time domain reflectometry, coupling losses, wave division multiplexing, dispersion and distortion in fibers and characteristics of fiber optic switches and couplers. As materials were developed they were tested on QCC students and then tested at a remote site, Suffolk County Community College (SCCC).

The model for our LFOT program can be applied to any technology. Our model expands access in three ways. First, students who would not commute daily to QCC, will be able to enroll in the LFOT program. Second, other institutions can develop their own programs, incorporating all or part of our LFOT courses via distance learning. Third, a group of institutions can use the model. Since many institutions would be sharing the equipment, the cost is spread out, access increased and the program affordable.

In this paper we will focus on the development of materials for the laboratory portions of the courses and their applicability to photonics education.

\section{DEVELOPMENT}

Laboratory exercises play a number of roles for science, engineering and technology students. The exercises illustrate and aid in the understanding of physical principles and help students develop skills in data analysis. Students are introduced to and learn to use modern instrumentation and develop "hands-on" skills. There are differences in emphasis for different group of students, for instance, technology students need to develop more "hands-on" skills, but all are necessary. Remote laboratories meet all the needs but that of developing "hands-on" skills. There is a fundamental difference between performing a laser alignment remotely and doing it by hand. And there is a necessity for technicians to be able to alignments by hand. Therefore, in developing a laboratory curriculum for distance learners, a method of providing the "hands-on" component must be included. We included the "hands-on" component by having the students come to our campus one or two full Saturdays a semester. There are other solutions for other situations. For instance, another college offering LFOT using our courses can provide the "hands-on" experience through low cost substitutions. For training alignment skills, a laser can be replaced by a cavity with a silvered mirror on one end, a partially reflecting mirror on the other end and some apertures and glass slides in between.

Just as for students who are physically present in the laboratory, distance learners must perform "real" experiments, acquire "real" data and run into "real" problems. For this to happen the distant learners must have control of the laboratory equipment, which requires the development of remote-controlled laboratories.

Laboratory exercises fit within two categories. Those that require the movement of objects of some sort, such as mirrors (Michelson interferometry), and those that only require the control of instrumentation (optical time domain reflectometry). Motorized mounts control all movements of devices, such as sensors, detectors and polarizers. These mounts are, in turn, controlled by an AutoOptics ${ }_{\mathrm{TM}}$ controller, which is interfaced with the computer. Integrated Photonics Systems supplies the AutoOptics ${ }_{\mathrm{TM}}$ controller, some motorized mounts, motors with adapters for assorted mounts and software, both Windows and LabVIEW $\mathrm{TM}_{\mathrm{TM}}$ versions.

\subsection{Laboratory manuals}

The laboratory manual must provide instructions for students who will be working without an instructor present. We have written an interactive lab manual, which includes simulated experiments as well as simulated instruments. Writeups contain video clips, still photos and diagrams of the experimental set-up along with simulations of the experiment. Included are simulations where the student works through the use of the software controls, step-by-step. In this way, the student becomes familiar with the "equipment" before performing the actual laboratory exercise.

When students work through the manual they must learn to control the equipment via computer. This requires the simulations in the manual to use the same control panels as are used in the exercise. For instruments controlled by

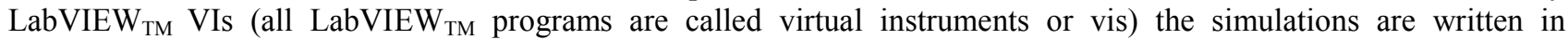
LabVIEW $_{\mathrm{TM}}$ and the front panels are identical to those used to control the actual laboratory equipment. The AutoOptics $_{\mathrm{TM}}$ control panel used most often is the Windows version, which makes writing a simulation more difficult. The simulation was written in Visual Basic (VB). The procedure was as follows. While the controller was being used, 
the pictures of the control panel were captured from the screen. This gave us a set of frames showing the control panel in each state of operation during the exercise, which was used to make a movie of the procedure and provide an instruction set. A VB form was overlaid on top of a picture of the controller with buttons simulating the action of the AutoOptics buttons. When a button is pressed a picture box produces the same result as the control panel does, figure 1.

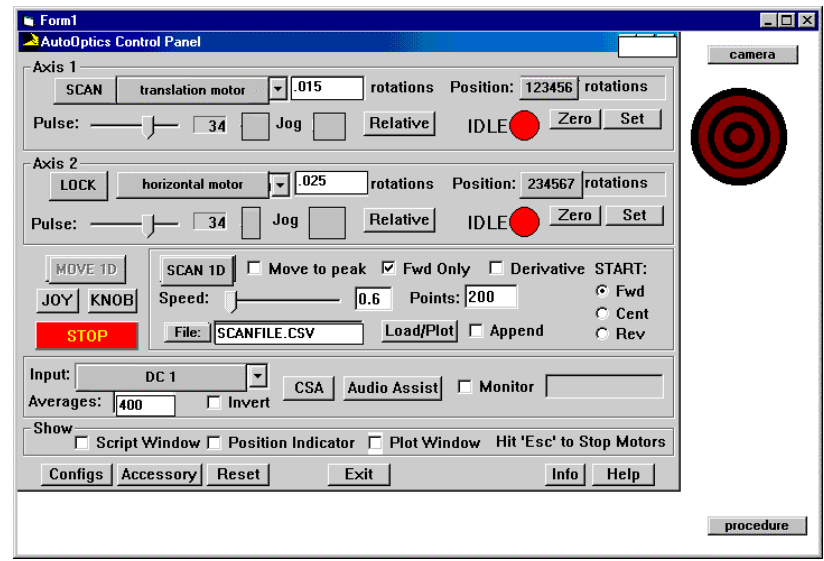

Figure 1a. AutoOptics simulation with fringe pattern.

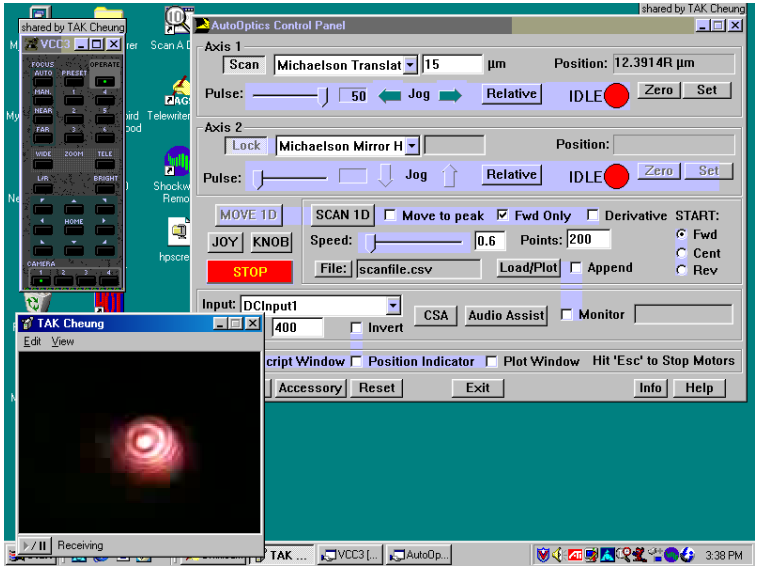

Figure 1b. Actual screen seen when performing exercise.

\subsection{Laboratory Exercises}

There are a number of components involved in the development of a set of laboratory exercises for distance learners. An easy solution used by some, is to have the remote student partner with students who are present in the laboratory classroom. This approach was unacceptable because the remote student controls nothing and only has as much input as his/her lab partners allow. Instead we chose to give remote students control of their own apparatus.

The development process started with a determination of which existing exercises should be converted for remote access. To that list was added those new exercises being developed to upgrade the curriculum. The exercises were then developed one at a time, with similar exercises developed in sequence, thereby reducing the development time for some exercises. For example, compare the exercises examining blazed diffraction gratings and the exercise measuring the dependence of reflectivity on angle of incidence and polarization. The apparatus for examining blazed gratings requires the rotation of the grating, the rotation of a detector, the translation of a detector to measure the incident power and the control of the laser wavelength. The apparatus for examining reflectivity as a function of polarization and angle of incidence requires all of the above except control of the laser wavelength and the addition of control of a half-wave plate to control polarization.

As with most laboratory exercises, ours had multiple objectives. The remote-controlled versions had to meet the objectives at least as well as their in-class counterparts. Objectives related to acquisition of "hands-on" skills could not be part of the remote exercises and were left for the sessions at QCC. As part of the set of Michelson interferometer exercises, students must align the interferometer. The original plan was to have remote students perform the alignment as well. After testing we determined that the coarse alignment of the interferometer was too difficult and time consuming to do remotely and that the coarse alignment skill is a valuable "hands-on" skill and should be part of the exercises performed at QCC. The fine alignment of the interferometer is part of the remote exercise.

The development continued with an outline of the procedure. It was at this point where we had to determine what equipment was required and how to control it. Determinations were made as to how much motion control was required. For example, the Michelson interferometer requires two motors to control the alignment of a one mirror and another motor to translate the other mirror, figure 2. For the interferometer, $3 \mathrm{~cm}$ of translation is adequate with a precision of better than $100 \mathrm{~nm}$.

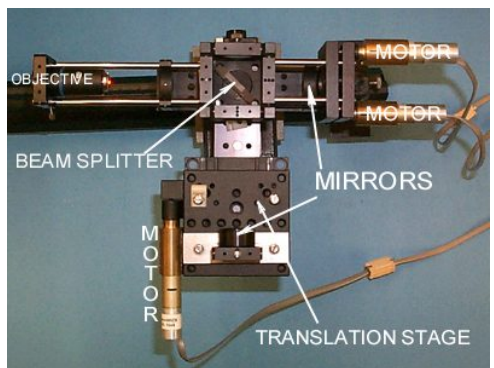

Figure 2 motorized Michelson interferometer 
The level of precision is the critical factor. We were able to use an AutoOptics controller with associated motors and stages because we did not require nanometer precision. The AutoOptics controller in combination with its software and data acquisition board allows for signals to be acquired through the controller. In this way any experiment which requires a signal as a function of position can be performed without any additional programming. The controller can control relays as well. Using the scripting language included with the controller, we developed an exercise to measure the index of refraction of a gas. A series of relays are used to turn on a vacuum pump and open a valve to evacuate a cell in one arm of the interferometer. The valve can be closed and another valve opened to allow gas into the cell. As the gas is bleeding into the cell the irradiance as a function of time at the pattern center is recorded, allowing the number of fringes to be counted as the cell returns to the initial pressure. From the length of the cell, the pressure difference and the number of fringes the index of refraction of the gas, at one atmosphere, is determined.

Instrumentation control was an issue as well. For each exercise we had to determine what instrumentation was required and how we would control it. When ever possible we used equipment on hand. We used three methods to control equipment. For instruments with dc outputs we used the AutoOptics controller and its associated data acquisition board, whenever possible. Otherwise, data acquisition was achieved through a data acquisition board controlled by LabVIEW. Some instruments, such as our Coherent Nd:YAG laser were equipped with serial (RS-232) control. LabVIEW vis were written to control them. The problem with serial instruments is that only one instrument can be control per computer serial port. Our first choice was to use equipment with a GPIB interface. The advantage of parallel communication is that up to 32 instruments can be controlled via a single port. We used LabVIEW vis to control these instruments. For many of these instruments LabVIEW drivers are available. In some cases, the driver fitted our needs. In other cases we modified the drivers because they either did meet our exact requirements or they had small bugs in them. And for some instruments the drivers provided were so problematic, that we wrote our own as we did in the cases where drivers were not available.

The last piece of the experimental apparatus is the same for every exercise. It is the computer controlled video camera with pan, zoom and tilt capabilities. Many of the exercises require the alignment of equipment. In order for remote students to perform alignments, they must be able to see what is happening. For example, in the exercises involving rotating a blazed grating or a glass block, the setting of zero degrees is critical. The students can accidentally reset the zero on the controller so that zero is improperly assigned. In order to fix their problem the students must rotate the device (grating, block or detector) for which zero is offset until the incident light reflects back on itself. The only way the students will know that the system is properly aligned is by viewing the moving laser spot.

\subsection{Remote access and control}

The completed exercises allowed for completed computer control. Computer control is not remote access. Many institutions including QCC have laboratories which incorporate computerized data acquisition and/or computer control of instrumentation. These labs are not necessarily accessible to remote students. Some method of remote access and control must be chosen. Before a decision was made on how the access is to be accomplished, we had to decide the conditions under which this access would occur. We wanted a system where we had control of who could access our laboratories and when. We wanted each lab group ( 2 students maximum) to have a specific assigned time. We did not want a system of passwords and we wanted to protect our instrumentation. Lastly, the access process should be relatively simple.

We chose to have the laboratory made accessible by remote sites through the internet, with the remote user sharing control of software running on the computer in the QCC lab, which is interfaced to all the equipment. The control of QCC equipment and software is limited in a number of ways. The connection is made using Microsoft's NetMeeting software. We chose NetMeeting because it is available free. Our laboratory is assigned a static IP address so remote users connect directly to our lab without having access to the QCC's network. With NetMeeting, only the specific applications chosen by us at QCC can be shared by the remote users. Another safeguard is to limit the amount of software loaded on the computer. As this computer will only be used for remote access only the appropriate software need be loaded, making corruption of the development software impossible. Once remote users have access and control, their range of control needs to be limited. We did not want them to be able to rewrite programs, control functions we made inaccessible or by equipment beyond preset ranges all of which is easily accomplished with LabVIEW. When using LabVIEW $_{\mathrm{TM}}$, control of instruments can be limited by password or by running front panels without diagrams. 
Since passwords can be discovered we opted for running front panels without diagrams. The diagram is the source code, so running a front panel without a diagram is equivalent to running an executable without the source code. Without the source code the program cannot be changed. The program only allows for specific inputs with specific ranges and can not be altered. For instance, the thermal lensing and second harmonic generation exercises require the ability to turn the lamps on and control the lamp current of a Nd:YAG laser. The front panel of the vi we wrote to control the Coherent $\mathrm{Nd}$ :YAG laser only contains a switch to turn the flashlamps on or off and a control for the flashlamp current. Without the ability to change the current limit, the student cannot exceed the preset maximum current value, thus protecting the laser.

\subsection{Testing}

Once initial development of materials and exercises was completed, testing was required to determine whether or not changes needed to be made. The first tests were by QCC students using the manual and perfroming the exercises in the lab room with the equipment and an instructor. Through these tests we found where the student needed help and made corrections to the manual as needed.

The next step was to test using the remote access. The first tests were with QCC students accessing the laboratory from a computer lab. There was an instructor available to help with any problems and determine if changes in procedure were required. The final tests were performed by students at Suffolk County Community College (SCCC). It was during thses tests that we realized the importantance of the audio connection via NetMeeting. Whenever the students ran into a problem they could just call out for help and the technician could respond. After incorporating suggestions from the remote students a final version was tested. The development of an exercise was considered completed when remote students could perform the exercise with only the interactive manual as a guide. We should note that the audio connection is critical when students are using the remote laboratory. If any problem with the equipment occurs as happens in any laboraotry setting, the students need a way to contact the technician.

\section{EXAMPLES}

We have completed the development of a number of exercises and are in the process of testing others. We will continue to expand the number of exercises. Exercises already completed include; using Michelson interferometers to measure the wavelengths of a tunable helium-neon $(\mathrm{HeNe})$ laser and the index of refraction of air, near field and far field diffraction, determining the polarization of a source, determining the characteristics of a blazed grating, determining the dependence of the reflectivity on the incident angle and polarization, studying optical activity, acousto-optics, electrooptics, second harmonic generation, thermal lensing, diode laser characteristics and optical time domain reflectometry. Exercises we are in the process of testing include; Q-switching, modelocking, laser threshold and slope efficiency and their dependence on loss and output coupler reflectivity, fiber coupling losses, and characteristics of fiber optic switches and couplers. We had developed an exercise on wave division multiplexing (WDM) and another on erbium-doped fiber amplifiers (EDFAs), but we have decided to make major changes in them. We are looking to develop a complete set of exercises around dense WDM and EDFAs in the near future.

One of the advantages of real exercises is that the students never know exactly what will happen. The same is true for the developers. We have demonstrated a number of these exercises at conferences ${ }^{2,3,4}$ and workshops and reported on them ${ }^{3,4,5}$. We will discuss some exercsies not reported on previously.

\subsection{Thermal Lensing}

In the thermal lensing exercise students determine the focal length of the laser rod in a Nd:YAG laser as a function of lamp current. The exercise consists of determining the position of the focus of a HeNe beam with the YAG lamps off, the object position, and the position of the focus of the HeNe beam with the lamps on, the image position. From the object and image positions the focal length can be determined if the rod is treated as a thin lens. This procedure is repeated for the lamps currents from the minimum to the maximum stipulated by the manufacturer of the laser. In order to determine the position of the focus of the HeNe beam the beam divergence has to be measured. This is done by scanning across the beam vertical finding the middle of the spot and then scanning horizontally to determine the width at one location and then repeating the measure some distance, generally one meter from the first. These measurements require a fiber (to give $50 \mu \mathrm{m}$ resolution) mounted such that it can be translated horizontally, vertically and longitudinally 
(along the direction of propagation of the beam), see figure 3. The motion is controlled by the AutoOptics controller and the dc output from the detector inputted through the controller. The laser lamps are controlled by a LabVIEW vi.

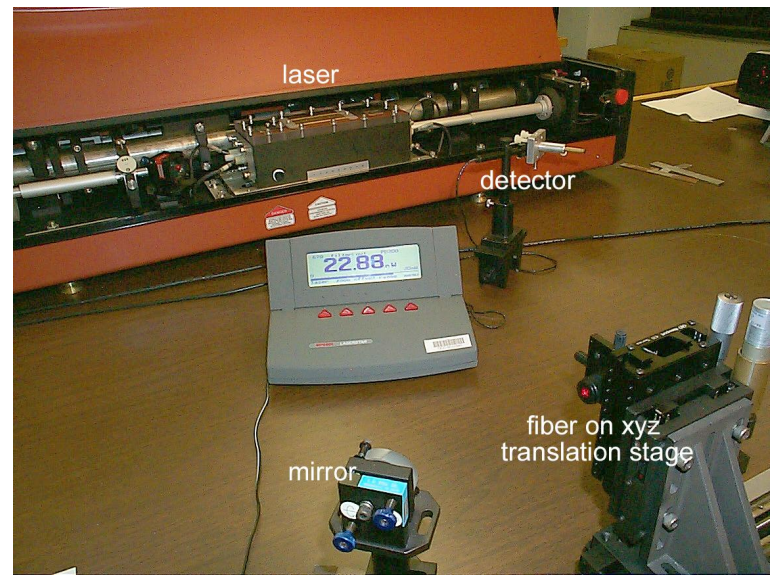

Figure 3a Actual apparatus for thermal lensing

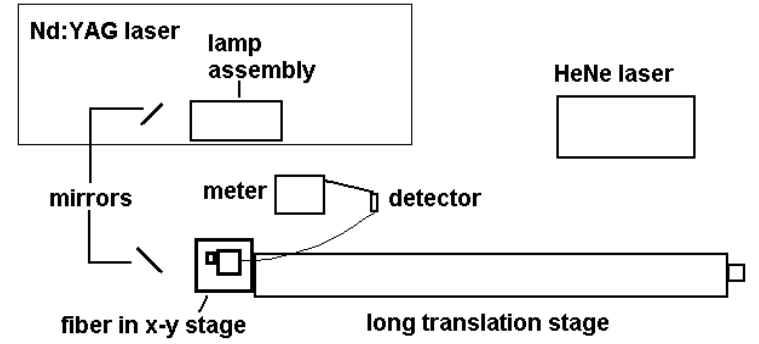

Figure $3 b$ Diagram of thermal lensing apparatus

After treating the rod as a thin lens, the more realistic approach of treating it as a thick lens is used. In order to determine the focal length of a thick lens, the principal planes must be located. For the focal plane and principal plane locations of a symetric thick lens to be determined, the image position must be determined for two distinct object positions. The second obect position is produced by inserting a lens into the HeNe beam by way of a translation stage.

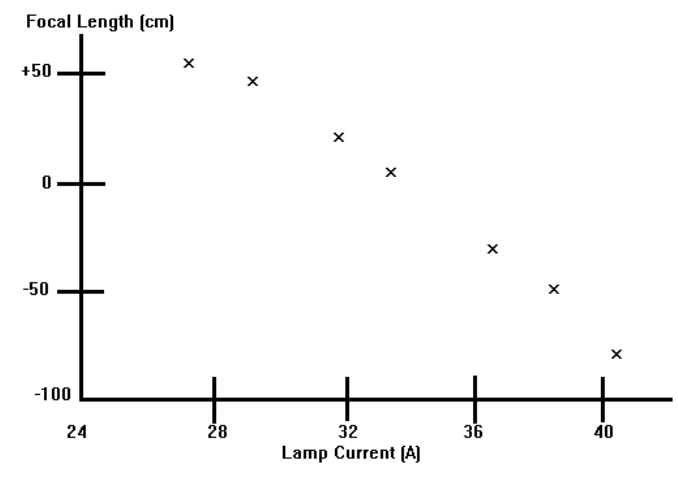

Figure 4 Focal length vs. lamp current for YAG laser rod
The focal length of the rod varies because the index of refraction of the rod material depends on temperature. As the lamp current increases the rod absorbs more energy, while being cooled by water flowing over the outside of the rod. Since the light from the flashlamps is focussed on the center of the rod, the student expectation is the rod will be hottest at the center and coolest at the edge and always act as a negative lens. Students do not consider how the absorption varies radially with lamp current. The actual results indicate that the focal length does vary with lamp current. The surprise for the students is the initial positive value of the focal length, figure 4 .

\subsection{Diode laser characteristics}

Diode lasers have become the most ubquitous in use, from sources in telecommunications to pumps in doped insulator solid state lasers. It is important for students to understand these lasers. We have developed a number of exercises on dioide laser characteristics that span two laboratory sessions.

The experimental apparatus is really two set-ups, figure 5. One consists of a diode lasers (wavelength of 780nm) in a thermoelectrical cooled mount without a collimating len, a fiber on an $x-y$ translation stage and a detector. The other consists of a diode laser in a thermoelectrically controlled mount with a collimating lens, a power meter detector on a translation stage, a monochromator and a detector. The diode laser controllers control both operating current and temperature. The motion of the translation stages is controlled by the AutoOptics controller. The signals from two of the detectors are collected via the AutoOptics controller. The monochromator and diode lasers are controlled by two LabVIEW vis, one for the lasers, one for the monochromator and the monochromator vi also collects the data from the detector connected to the monochromator. 

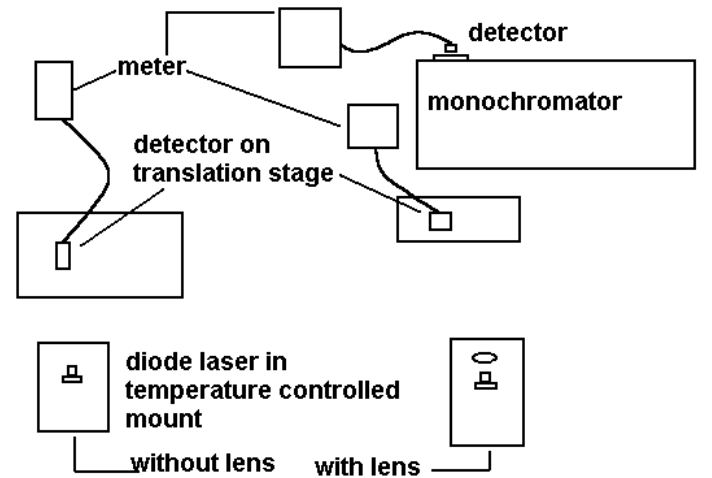

The exercises consist on determining the beam divergence of the diode laser, determining the laser threshold and slope as a function of temperature, the mode structure as a function of current and temperature and the wavelenght as a function of current and temperature.

The beam divergence is measure by scanning a detector with a small aperature across the beam both vertically and horizontally at a fixed distance from the laser. The beam width is determined from the scans (figure 6 ) and the beam divergence calculated.

Figure 5 Diode laser apparatus
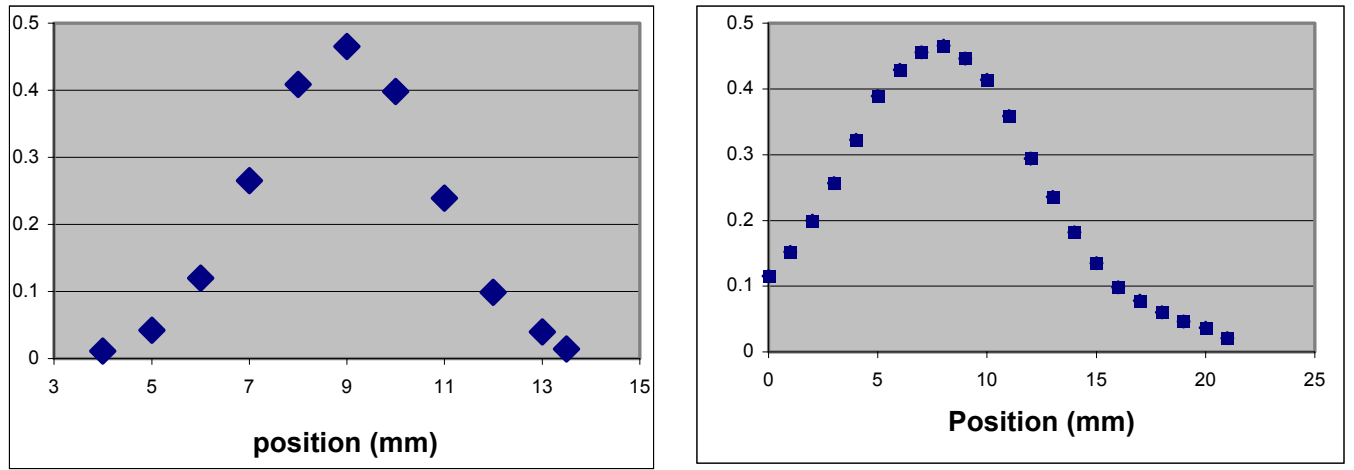

Figure 6 Diode laser beam profiles, horizontal and vertical

The laser threshold and slope of power versus current are measured by moving the power meter detector into the path of the laser beam and measuring the power at different operating currents while holding the temperature fixed for a number of different operating temperatures. The threshold and slope are determined from the power versus current curves, figure 7. The wavelength as a function of temperature, with current fixed and as a function of current with temperature

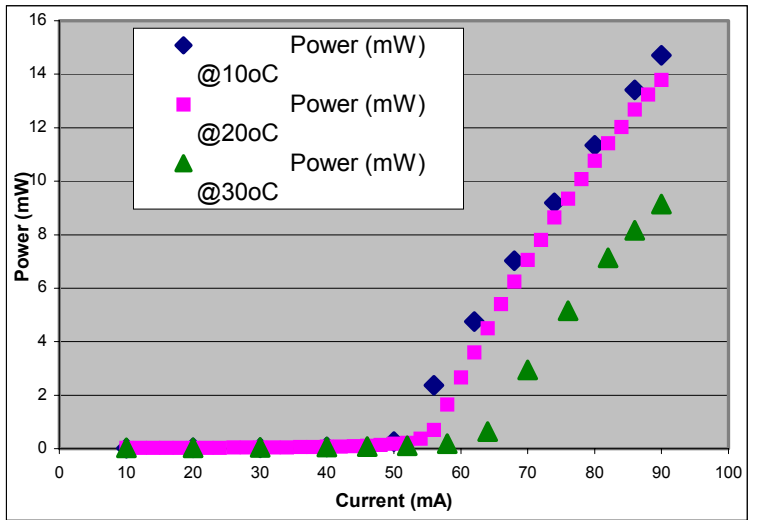

Figure 7 Laser power vs. current

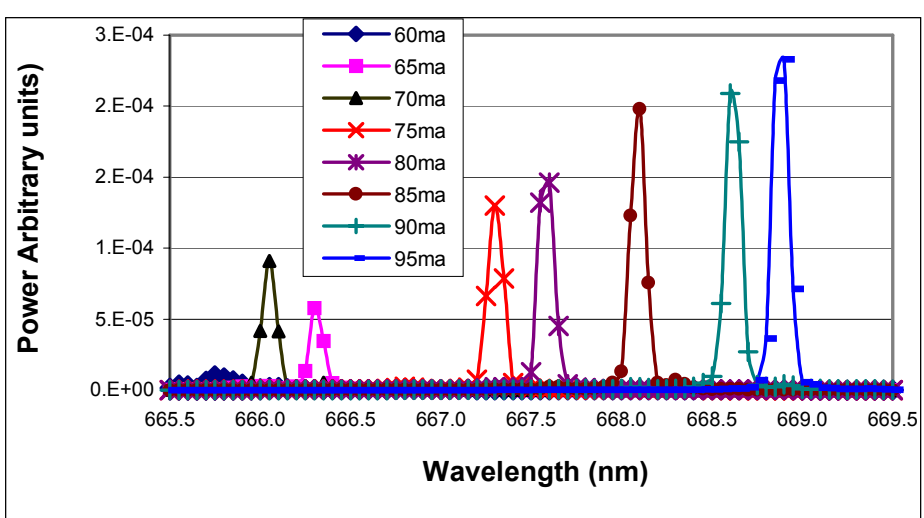

Figure 8 Diode laser spectra at various currents

fixed is determined by scanning the monochromator at each current and temperature and determining the peak wavelength from the curves, figure 8 . These curves allow the student to observe the transition from LED emission, 


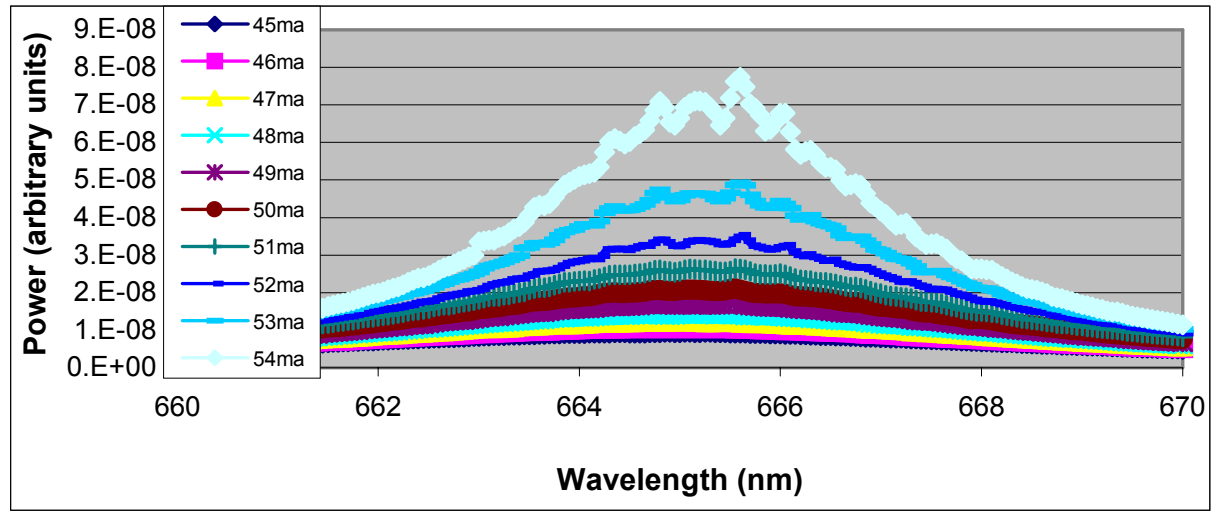

figure 9 to laser emission, figure $10 . \quad$ The peak wavelength as a function of temperature or current is then plotted, figure 11. These curves also show the mode structure of the laser output, figure 12 .

Figure 9 Diode laser spectra below threshold

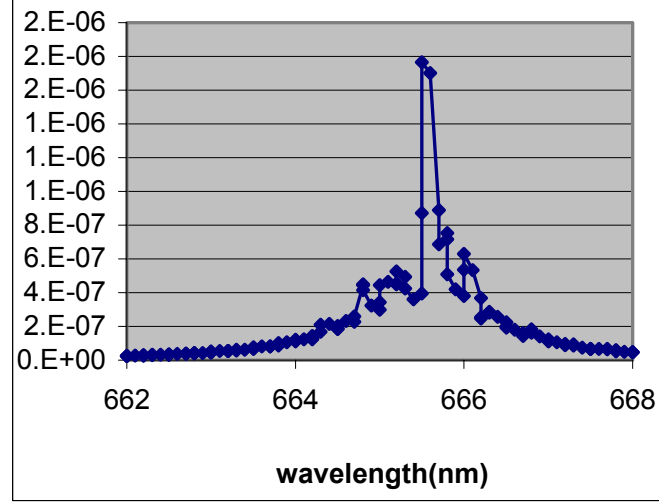

Figure 10 Diode laser spectrum just above threshold

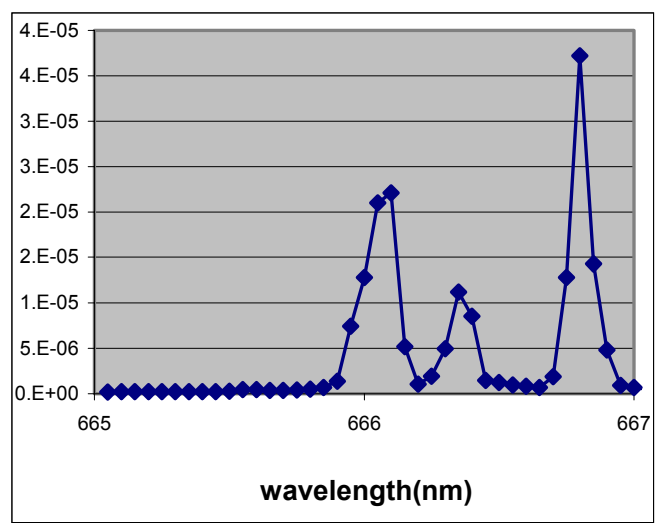

Figure 12 Laser spectrum at $67 \mathrm{ma}$ drive current
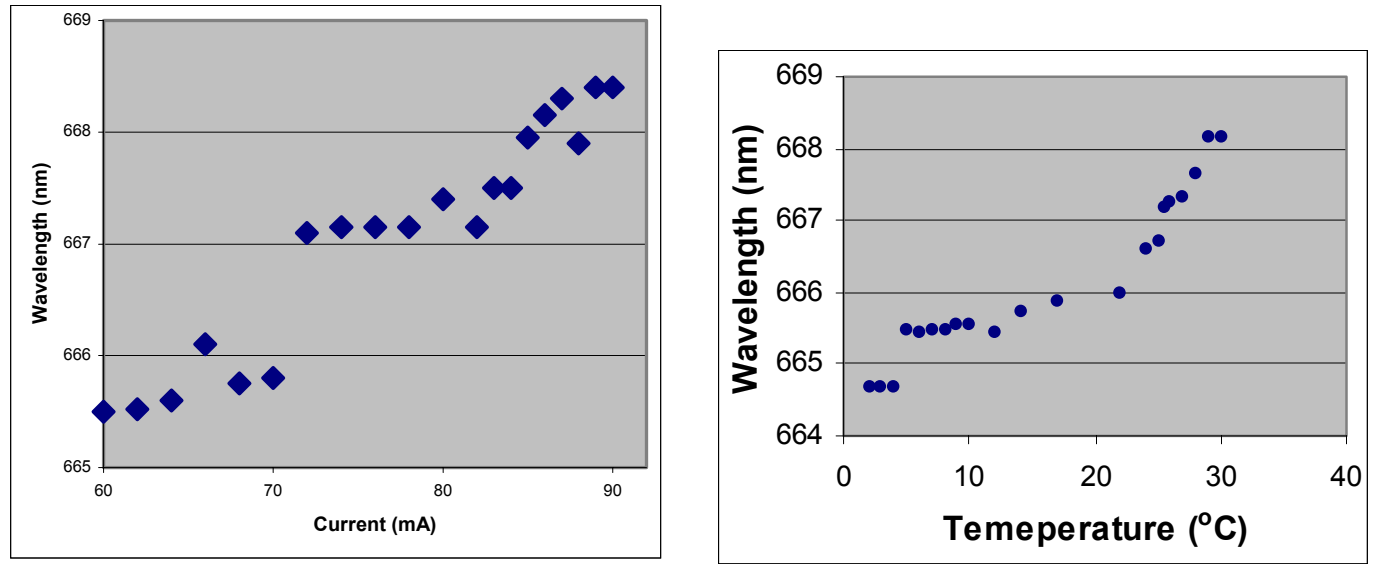

Figure 11 Peak wavelength vs. current for fixed temperature and peak wavelength vs. temperature for fix current

What is interseting about the lasers we used is that they do not exactly follow the curves on the spec sheets. As the current increases the peak wavelength of a specific mode increases, as expected, but the distribution of irradiance between modes varies and the laser does not make a transition from multimode operation to single mode operation. The general rule of the higher the current the smaller the number of modes does not apply except near threshold when the laser goes from operating at many modes to operating at a few modes. Another interesting behavior is at certain currents 
the laser wavelength decreases instead of increases with increasing current by mode-hopping in the "wrong" direction, something the students do not expect.

\section{STUDENT PERFORMANCE AND CONCLUSIONS}

\subsection{Student performance}

The SCCC students who performed the remote laboratories demonstrated superior understanding of the material both through lab reports and quizzes. From discussions these students indicated that the fact that they were on their own required them to spend more time preparing for the laboratory session. The students enjoyed the laboratory sessions because they felt they had freedom to make changes in the procedure without someone looking over their shoulder. They also appreciated the real nature of the experiments although they occasionally were frustrated when they made an error and had to figure out how to correct it. QCC students seem to benefit as well. They felt the changes made in the exercises due to the development of the remote exercises were positive and made their laboratory class more interesting.

\subsection{Conclusions}

Remote-controlled photonics laboratories are a viable method for providing access to distance learners. The remote laboratory experience is in many ways as rich as the traditional in-class situation. The development of computercontrolled remote laboratory exercises helps to improve the laboratory experience for all students through the upgrading and improving of existing exercises. Although there are indications that remote laboratories improve student performance, the sample size is as yet too small to justify that conclusion.

Remote-controlled laboratory apparatus can be used for laboratory experiments. We differentiate between experiments and exercises in the following way. An exercise has a specific teaching outcome associated with it and is perform many times by many students. An experiment is a situation where the outcome is unknown both to the student and the instructor. They may have a good idea based on some previous work but the specific experiment was not done before.

The skills required for the development of remote-controlled laboratory exercises can be used to computerize laboratory equipment used in research. Remote-controlled laboratories can provide access to research facilities at a distance providing for greater access and collaboration.

\section{References}

1. Navarra, A., Hull, D., Guenther, A. and Pedrotti, L. "Whence the Technicians", Photonics Spectra, 35(4), 115-118, 2001

2. Lieberman, D. "Remote-Controlled Laboratory Demonstration", Report on the Fall 1999 Meeting of TYC21, Region 15, October 1999

3. Lieberman, D. and Cheung, T., "Distance Learning and Remote-Controlled Laboratories", Abstract, Conference on Computing in the Disciplines, March 2000.

4. Lieberman, D. and Cheung, T., "Distance Learning and Remote-Controlled Laboratories for Photonics Technology", Proceedings of the American Society for Engineering Education, Annual Conference, June 2001.

5. Lieberman, D. and Cheung, T.," Distance Learning and Remote-Controlled Laboratories for Photonics" Technology, Proceedings of the American Society for Engineering Education, Middle Atlantic Regional Conference, April 2000. 